\title{
Ant-Colony Based Near-ML Space-Time Multiuser Detection for the STBC Assisted DS-CDMA Uplink
}

\author{
Chong Xu, Lie-Liang Yang and Lajos Hanzo \\ School of ECS., Univ. of Southampton, SO17 1BJ, UK. \\ Tel: +44-23-80-593 125, Fax: +44-23-80-593 045 \\ Email: \{cx05r, lly, lh $\} @$ ecs.soton.ac.uk, http://www-mobile.ecs.soton.ac.uk
}

\begin{abstract}
In this contribution, a novel Ant Colony Optimization (ACO) based Space-Time (ST) Multiuser Detection (MUD) algorithm was proposed in order to improve the Bit Error Ratio (BER) versus Signal-to-Noise Ratio (SNR) performance achieved by the Space-Time Block Code (STBC) assisted two transmit antenna aided fully loaded DS-CDMA system directly employing an ACO based MUD algorithm. Our simulation results demonstrate that the proposed ACO based MUD algorithm enables the fully loaded system to approach the single user performance associated with a second-order diversity gain. Furthermore, the complexity of the improved ACO based MUD algorithm is a factor of $10^{18}$ lower than that of the Maximum Likelihood (ML) MUD, when $K=32$ users are supported by the STBC $\mathcal{G}_{2}$ asssited DS-CDMA systems employing Gold codes having a length of 31 chips.
\end{abstract}

\section{INTRODUCTION}

Ant Colony Optimization (ACO) was inspired by studying the foraging behavior of ants. It was first invoked by Colorni, Dorigo and Maniezzo [1] in 1991. This population-based approach has recently been applied to a large number of so-called Non-deterministic Polynomial (NP)-hard combinatorial optimization problems [1]-[5]. These investigations motivated its employment in near-Maximum Likelihood (ML) Multi-User Detection (MUD) aided Multiple Access (MA) systems [6]-[9], [11], [12].

The ACO based MUD of both [9], [11], [12] and of [6]-[8] are capable of achieving a lower BER at a lower complexity, than the Genetic Algorithm (GA)-based MUDs of [13]-[15]. The authors of [11], [12] achieved a near-ML BER performance in the context of various Multi-Carrier (MC) DS-CDMA systems employing 31chip Gold codes as the Time Domain (TD) spreading sequence, while supporting $K=32$ users. Although it is not explicitly shown here owing to lack of space, we found that the required number of FLoating point Operations Per Second (FLOPS) was a factor of $10^{8}$ lower than that of the ML MUD.

Against this background, a novel system scenario is proposed in Sec. II in order to investigate potential techniques of improving the ACO based MUD algorithm of [12]. In the system scenario investigated in this paper, two transmit antennas are used by each Mobile Station (MS) to construct a Space-Time Block Code (STBC) [16], [17]. At the carrier frequency of $2 \mathrm{GHz}$, the wavelength is $15 \mathrm{~cm}$ and hence the afforded antenna-separation at the shirt-pocket-sized MS is insufficient to ensure independent fading at the BS, but fortunately the antenna-separation at a laptop may reach $10 \lambda$, which is indeed sufficiently high to satisfy the independent fading condition [10].

However, the BER performance of the ACO based MUD algorithm of [11], [12] exhibits an error-floor. Against this background, in this contribution, a novel ACO based MUD algorithm is proposed for mitigating the error floor.

The outline of the paper is as follows. The system's architecture is detailed in Section II, while Section III details both the traditional ACO-aided MUD algorithm applied in the STBC assisted DS-CDMA system and the novel improved ACO-aided MUD algorithm for the proposed system. Our simulation results detailed in Section IV

Acknowledgements: The financial support of the EPSRC, UK; of the Vodafone DHPA, and that of the EU is gratefully acknowledged. demonstrate the success of the proposed algorithm in improving the ACO based MUD of [11], [12], despite operating at a low complexity. Our conclusions are provided in Section V.

\section{SySTEM DESCRIPTION}

In each user's transmitter, the bit stream is firstly space-time block coded according to the $\mathcal{G}_{2}$ principle [17]. The signals $v_{k 1}$ and $v_{k 2}$ are input to the two $\mathcal{G}_{2}$ branches within the first symbol duration, while during the second symbol interval, $-v_{k 2}^{*}$ and $v_{k 1}^{*}$ are transmitted. Both branches of symbols will be spread using the same CDMA spreading code $\mathbf{c}_{k}$ having a length of $N_{s}$ chips, which is assigned by the BS to the $k$ th user. Then the signals are transmitted from the two antennas via the independently faded non-dispersive Rayleigh channels $h_{k 1}$ and $h_{k 2}$ respectively, for all the $k=1,2, \ldots, K$ users. The real and imaginary parts of the complex-valued Gaussian distributed fading envelope have a mean of zero and a variance of $1 / 2$.

Finally, during each of the $N_{s}$ chip intervals of the two symbol durations, the composite multiuser signal is given by the sum of all the symbols transmitted from the $2 K$ antennas of the $K$ users via the $2 K$ channels plus the Additive White Gaussian Noise (AWGN). The AWGN encountered during the first and the second symbol duration can be encapsulated in the $\left(N_{s} \times 1\right)$-element vectors $\mathbf{n}_{1}$ and $\mathbf{n}_{2}$, respectively. Each element $n_{i n_{s}}$, for $i=1,2$ and $n_{s}=1,2, \ldots, N_{s}$ is an instantaneous value of a complex-valued Gaussian random variable with a zero-mean and a variance of $2 \sigma_{n}^{2}$. Hereby, the $\left(N_{s} \times 1\right)$ element signal vector $\mathbf{r}_{1}$ and $\mathbf{r}_{2}$ containing the signals received during the $N_{s}$ chip intervals in the first and the second symbol duration are quantified by $\mathbf{r}_{1}=\xi \sum_{k=1}^{K} \mathbf{c}_{k}\left[h_{k, 11} v_{k 1}+h_{k, 21} v_{k 2}\right]+\mathbf{n}_{1}$, $\mathbf{r}_{2}=\xi \sum_{k=1}^{K} \mathbf{c}_{k}\left[h_{k, 21} v_{k 1}^{*}-h_{k, 11} v_{k 2}^{*}\right]+\mathbf{n}_{2}$, where $\xi=1 / \sqrt{2 N_{s}}$ is the energy of the signal transmitted from each antenna within every chip-duration.

The transmitted signal vector $\mathbf{v}_{k}=\left[v_{k 1}, v_{k 2}\right]^{T}$ contains the original symbols $v_{k 1}$ and $v_{k 2}$ of the $k$ th user. In order to generate the received signal vector, firstly the $\left(N_{s} \times 1\right)$-element signal vector $\mathbf{r}_{2}$ received during the second symbol duration is Hermintian transposed, yielding $\mathbf{r}=\left[\mathbf{r}_{1}^{T}, \mathbf{r}_{2}^{H}\right]^{T}$. Then the estimated complex-valued Channel Impulse Response (CIR) taps associated with the two antennas are used to construct the channel-matrix of

$$
\mathbf{H}_{k}=\left[\begin{array}{cc}
h_{k 1} & h_{k 2} \\
h_{k 2}^{*} & -h_{k 1}^{*}
\end{array}\right] .
$$

Thirdly, the $\left(N_{s} \times 1\right)$-element AWGN vector $\mathbf{n}_{2}$ encountered during the second symbol duration is also Hermitian transposed to form $\mathbf{n}=\left[\mathbf{n}_{1}^{T}, \mathbf{n}_{2}^{H}\right]^{T}$.

Therefore, the $\left(2 N_{s} \times 1\right)$-element received signal vector $\mathbf{r}$ can be expressed as:

$$
\mathbf{r}=\xi \sum_{k=1}^{K} \mathbf{C}_{k} \mathbf{H}_{k} \mathbf{v}_{k}+\mathbf{n}=\xi \mathbf{C H} \mathbf{v}+\mathbf{n},
$$


where we have

$$
\begin{aligned}
\mathbf{C} & =\left[\mathbf{C}_{1}, \mathbf{C}_{2}, \ldots, \mathbf{C}_{K}\right] \\
\mathbf{H} & =\operatorname{diag}\left\{\mathbf{H}_{1}, \mathbf{H}_{2}, \ldots, \mathbf{H}_{K}\right\} \\
\mathbf{v} & =\left[\mathbf{v}_{1}^{T}, \mathbf{v}_{2}^{T}, \ldots, \mathbf{v}_{K}^{T}\right]^{T} .
\end{aligned}
$$

\section{Ant-Colony Optimization Aided Space-Time MUD}

\section{A. Traditional ACO Aided ST-MUD}

The MUD's goal is to identify the $2 K$-element ML data-vector of the twin-antenna-aided $K$ users at the BS with a high probability, despite searching through only a small fraction of the $2^{2 K}$-element search-space.

TABLE I: The $(2 \times 2 K)$-element route table of the multi-user STBC

\begin{tabular}{|c|c|c|c|c|c|c|c|}
\hline$s_{i}^{(1)}$ & $j=1$ & $j=2$ & $j=3$ & $j=4$ & & $j=2 K-1$ & $j=2 K$ \\
\hline+1 & $v_{11}=s_{1}^{(1)}$ & $v_{12}=s_{1}^{(1)}$ & $v_{21}=s_{1}^{(1)}$ & $v_{22}=s_{1}^{(1)}$ & . & $v_{K 1}=s_{1}^{(1)}$ & $v_{K 2}=$ \\
\hline & $v_{11}=s_{2}^{(1)}$ & $v_{12}=s_{2}^{(1)}$ & $v_{21}=s_{2}^{(1)}$ & $v_{22}=s_{2}^{(1)}$ & $\cdots$ & $v_{K 1}=s_{2}^{(1)}$ & $v_{K 2}=$ \\
\hline
\end{tabular}
decoder employing the ACO principle of [11], [12], when the system supports $K$ users and each MS is equipped with two transmit antennas.

1) Route Table: The interpretations of the number of rows and columns associated with the $(2 \times 2 K)$-element route table are the same, as in the MC DS-CDMA system of [11]. More explicitly, the rows in Tab. I represent the legitimate BPSK modulated +1 and -1 symbols, while the columns of the route-table are the $2 K$-element twin-antenna symbols of the $K$ users. In terms of ACO parlance, the two rows of the table provide the two options for an artificial ant to consider during its passage through the set of legitimate $2 K$ element data vectors. In Tab. I, the legitimate symbols +1 and -1 are represented by $s_{1}^{(1)}$ and $s_{2}^{(1)}$, respectively.

The symbol $\mathbf{s}_{i}^{(m)}$ is introduced in order to represent the $i$ th legitimate BPSK symbol combination in the full set containing all the $\mathcal{M}=2^{m} m$-bit symbol combinations. The index $i$ is defined as the decimal interpretation of the binary symbol vector plus one. The binary symbol vector is obtained by assigning +1 to 0 and -1 to 1 . The symbol in the $j$ th column and $i$ th row of the route table is denoted by $b_{j i}=s_{i}^{(1)}$, for $j=1, \ldots, 2 K$ and $i=1,2$.

2) Cellular Likelihood: The cellular likelihood $\mathfrak{l}\left(b_{j i}\right)$ represents the likelihood that the BPSK symbol $b_{j i}$ was transmitted as the $j$ th element of the $2 K$-element vector, which corresponds to the $j$ th row and the $i$ th column of the $(2 \times 2 K)$-element route table adopted by the traditional hard-output ACO algorithm, which may be formulated as [9]:

$$
\begin{gathered}
\mathfrak{l}\left(b_{j i}\right)=2 \Re\left\{\xi b_{j i} y_{j}\right\}-\xi^{2} b_{j i} R_{j j} b_{j i}, \\
j=1,2, \ldots, 2 K ; \quad i=1,2 ;
\end{gathered}
$$

where $y_{j}$ is the $j$ th element of the $(2 K \times 1)$-element matched filter output vector $\mathbf{y}$ given by

$$
\begin{aligned}
\mathbf{y} & =\mathbf{H}^{H} \mathbf{C}^{T} \mathbf{r}=\xi \mathbf{H}^{H} \mathbf{C}^{T} \mathbf{C H} \mathbf{v}+\mathbf{H}^{H} \mathbf{C}^{T} \mathbf{n} \\
& =\xi \mathbf{R} \mathbf{v}+\tilde{\mathbf{n}} .
\end{aligned}
$$

Furthermore, $\mathbf{R}$ in Eq.(6) is the $(2 K \times 2 K)$-element correlation matrix of the composite matrix $\mathbf{C H}$, where we have

$$
\begin{aligned}
\mathbf{R} & =\mathbf{H}^{H} \mathbf{C}^{T} \mathbf{C H} \\
& =\left[\begin{array}{cccc}
\boldsymbol{\Sigma}_{1} & \rho_{12} \boldsymbol{\Lambda}_{12} & \cdots & \rho_{1 K} \boldsymbol{\Lambda}_{1 K} \\
\rho_{12} \boldsymbol{\Lambda}_{21} & \boldsymbol{\Sigma}_{2} & \cdots & \rho_{2 K} \boldsymbol{\Lambda}_{2 K} \\
\vdots & \vdots & \ddots & \vdots \\
\rho_{1 K} \boldsymbol{\Lambda}_{K 1} & \rho_{2 K} \boldsymbol{\Lambda}_{K 2} & \cdots & \boldsymbol{\Sigma}_{K}
\end{array}\right]
\end{aligned}
$$

and $\boldsymbol{\Sigma}_{k}=\left(\sum_{i=1}^{2}\left|h_{k i}\right|^{2}\right) \mathbf{I}_{2}$, while $\boldsymbol{\Lambda}_{k j}=\mathbf{H}_{k}^{H} \mathbf{H}_{j}$ for $k=$ $1, \ldots, K, j=1, \ldots, K$ and $j \neq k$. Finally, $\mathbf{I}_{2}$ represents a $(2 \times 2)$ element identity matrix and the correlation coefficient $\rho_{k j}$ between $\mathbf{c}_{k}$ and $\mathbf{c}_{j}$ is given by $\rho_{k j}=\mathbf{c}_{k}^{T} \mathbf{c}_{j} / N_{s}=\sum_{n_{s}=1}^{N_{s}} c_{k n_{s}} c_{j n_{s}} / N_{s}$, for $k=1, \ldots, K, j=1, \ldots, K$ and $j \neq k$.

Therefore, $R_{j j}$ in Eq.(6) is the $j$ th diagonal element of $\mathbf{R}$, whose value is $\sum_{i=1}^{2}\left|h_{k i}\right|^{2}$.

3) Vector Likelihood: The likelihood of a particular $(2 K \times 1)$ element $K$-user twin-antenna vector $\mathbf{s}_{i}^{(2 K)}$ in the full set $\mathbb{S}^{(2 K)}$ containing all the $2^{2 K}$ vectors is given by

$$
\begin{array}{r}
\mathfrak{L}\left(\mathbf{s}_{i}^{(2 K)}\right)=2 \Re\left\{\xi \mathbf{s}_{i}^{(2 K) T} \mathbf{y}\right\}-\xi^{2} \mathbf{s}_{i}^{(2 K) T} \mathbf{R s}_{i}^{(2 K)}, \\
i=1,2, \ldots, 2^{2 K}
\end{array}
$$

where $\mathbf{s}_{i}^{(2 K)}$ is the route followed by one of the $\zeta$ ants in the routetable, which is formed by concatenating the $2 K$ passages chosen by that particular ant, yielding:

$$
\begin{aligned}
\mathbf{s}_{i}^{(2 K)}=\left[\begin{array}{llll}
s_{\imath_{1}}^{(1)} & s_{\imath_{2}}^{(1)} & \cdots & s_{\imath_{2 K}}^{(1)}
\end{array}\right]^{T} \\
\forall j=1, \ldots, 2 K: \imath_{j} \in\{1,2\} .
\end{aligned}
$$

4) Traditional Algorithm of Determining N: In the benchmarker ACO-aided ST-MUD algorithm characterized by Alg. 1, both the probability matrix $\mathbf{p}$ and the integer matrix $\mathbf{N}$ have the same size as the $(2 \times 2 K)$-element route table. Each element of the matrix $\mathbf{N}$ represents the number of ants choosing the corresponding entry in the route-table. For each of the $2 K$ columns in the route-table, the sum of the numbers of ants choosing the two entries should be equivalent to the total number of artificial ants $\zeta$ adopted by the algorithm, yielding $N_{j 1}+N_{j 2}=\zeta$, for $j=1, \ldots, 2 K$.

Firstly, $N_{j i_{1}}$ number of ants choose the route table entry associated with the higher probability, where $N_{j i_{1}}$ is calculated as the rounded value of the product of $\left(\zeta \times p_{j i_{1}}^{\prime}\right)$. Similarly, $N_{j i_{2}}$ represents the number of ants, which choose the other route table entry, namely that associated with the lower probability, where we have $N_{j i_{2}}=$ $\zeta-N_{j i_{1}}$.

The interpretations of the 'sort' function and ' $\imath$ ' function of Alg. 1 are provided in the Appendix. The ACO algorithm portrayed above is

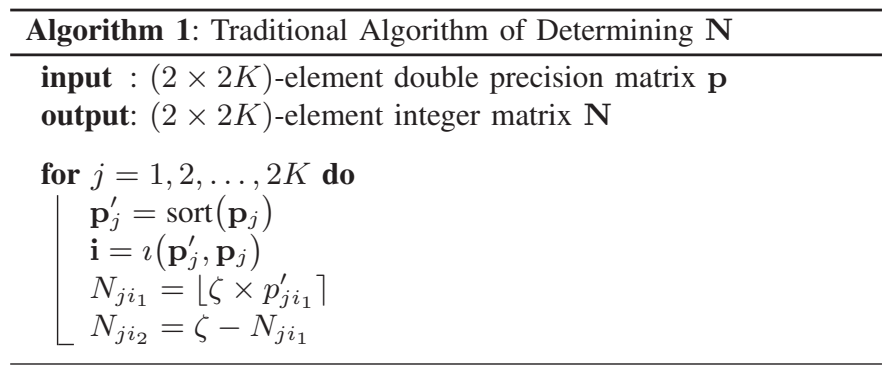

then embedded into the STBC MUD and the achievable performance of the system considered is characterized in Fig. 1. As observed in Fig. 1, the BER versus SNR curve of the system employing the ACOaided hard-decision STBC MUD exhibits an error-floor. Hence, in the next section we will propose a novel ACO-aided MUD algorithm, which will enable the fully-loaded system supporting as many users as the number of chips in the spreading sequence to approach the BER performance achieved by the single-user system.

\section{B. Improved ACO aided ST-MUD}

In this section, novel ACO-based hard-output ST-MUD algorithm is proposed in order to provide an improved BER performance at the cost of a moderate complexity increase. To elaborate a little further, the proposed algorithm is different from the traditional ACO based 
MUD algorithm, as it has been stated in Subsection III-A in three aspects, which are the size of the route table, the formula derived for calculating the cellular likelihood of each cell and the algorithm used for calculating the integer matrix $\mathbf{N}$ of $\mathrm{Alg}$. 1. For the sake of completeness, we briefly describe the above three aspects as well as the evaluation of the vector likelihood adopted by the proposed ACO based MUD algorithm.

TABLE II: $(4 \times K)$-element route table of the MU STBC decoder employing the improved ACO algorithm, when the system supports $K$ users and each MS is equipped with two transmit antennas.

\begin{tabular}{|c|c|c|c|c|}
\hline $\mathbf{s}_{i}^{(2) T}$ & $\begin{array}{c}j=1 \\
\mathbf{v}_{1}=\left[\begin{array}{ll}v_{11} & v_{12}\end{array}\right]^{T}\end{array}$ & $\begin{array}{c}j=2 \\
\mathbf{v}_{2}=\left[\begin{array}{ll}v_{21} & v_{22}\end{array}\right]^{T}\end{array}$ & $\begin{array}{l}\cdots \\
\cdots\end{array}$ & $\begin{array}{c}j=K \\
\mathbf{v}_{K}=\left[\begin{array}{ll}v_{K 1} & v_{K 2}\end{array}\right]^{T}\end{array}$ \\
\hline $1[+1+1]$ & $\mathbf{v}_{1}=\mathbf{s}_{1}^{(2)}$ & $\mathbf{v}_{2}=\mathbf{s}_{1}^{(2)}$ & $\ldots$ & $\mathbf{v}_{K}=\mathbf{s}_{1}^{(2)}$ \\
\hline $2\left[\begin{array}{ll}+1 & -1\end{array}\right]$ & $\mathbf{v}_{1}=\mathbf{s}_{2}^{(2)}$ & $\mathbf{v}_{2}=\mathbf{s}_{2}^{(2)}$ & $\cdots$ & $\mathbf{v}_{K}=\mathbf{s}_{2}^{(2)}$ \\
\hline $3[-1+1]$ & $\mathbf{v}_{1}=\mathbf{s}_{3}^{(2)}$ & $\mathbf{v}_{2}=\mathbf{s}_{3}^{(2)}$ & $\cdots$ & $\mathbf{v}_{K}=\mathbf{s}_{3}^{(2)}$ \\
\hline $4\left[\begin{array}{ll}-1 & -1\end{array}\right]$ & $\mathbf{v}_{1}=\mathbf{s}_{4}^{(2)}$ & $\mathbf{v}_{2}=\mathbf{s}_{4}^{(2)}$ & $\ldots$ & $\mathbf{v}_{K}=\mathbf{s}_{4}^{(2)}$ \\
\hline
\end{tabular}

1) Route Table: The route table in the proposed ACO based STMUD algorithm has $(4 \times K)$-element, where we have $m=2$. More explicitly, the four entries of each column correspond to the $(\mathcal{M}=$ $\left.2^{m}=4\right)$ legitimate $(m=2)$-symbol combinations, as indicated in Tab. II, which should be contrasted to Table I.

2) Cellular Likelihood: As shown in Subsec. III-A, for $m=2$ the submatrix $\mathbf{R}_{j j}^{(2)}, j=1, \ldots, K$ is a diagonal matrix. Therefore, the cellular likelihood $\tilde{\mathfrak{l}}_{j i}$ of the $(i, j)$ th cell can be expressed as

$$
\begin{aligned}
\tilde{\mathfrak{l}}_{j i}=2 \Re\left\{\mathbf{s}_{i}^{(2) T} \mathbf{y}_{j}^{(2)}\right\}-\xi^{2} \mathbf{s}_{i}^{(2) T} \mathbf{R}_{j j}^{(2)} \mathbf{s}_{i}^{(2)} \\
=\mathfrak{l}_{\varphi_{j \imath_{1}}}+\mathfrak{l}_{\psi_{j} \imath_{2}}, \quad \imath_{1}, \imath_{2} \in\{1,2\} \\
\quad \forall j=1,2, \ldots, K \text { and } i=1,2,3,4 ;
\end{aligned}
$$

subject to $\varphi_{j}=(2 j-1), \psi_{j}=2 j$ and $\mathbf{s}_{i}^{(2)}=\left[\begin{array}{ll}s_{i_{1}}^{(1)} & s_{i_{2}}^{(1)}\end{array}\right]^{T}$.

3) Vector Likelihood: The formula quantifying the vector likelihood adopted by the improved ACO based ST-MUD algorithm remains the same as in Eq. (9), which is the one adopted by the traditional ACO based ST-MUD algortihm. The only difference lies in the formation of the $(2 K \times 1)$-element candidate vector $\mathbf{s}_{i}^{(2 K)}$ that concatenates all the $K$ number of 2-by-1 passages pursued by the same ant, yielding

$$
\begin{aligned}
\mathbf{s}_{i}^{(2 K)}= & {\left[\begin{array}{llll}
\mathbf{s}_{\imath_{1}}^{(2)} & \mathbf{s}_{\imath_{2}}^{(2)} & \cdots & \mathbf{s}_{\imath_{K}}^{(2)}
\end{array}\right]^{T} } \\
& \forall j=1, \ldots, K: \imath_{j} \in\{1,2,3,4\} .
\end{aligned}
$$

4) Improved Algorithm of Determining $\mathbf{N}$ : Both the probability matrix $\mathbf{p}$ and the integer matrix $\mathbf{N}$ have the same size as the $(4 \times K)$ element route table. However, the taditional MUD shown in Alg. 1 may become unable to provide an integer-valued matrix $\mathbf{N}$ having reasonable entries for the improved ACO based ST-MUD algorithm. For example, assume that the probabilities of choosing the four entries associated with the $j$ th column, $j=1,2, \ldots, K$ are given by the probability vector $\mathbf{p}_{j}=[0.36,0.27,0.15,0.22]$, and $\zeta=10$ artificial ants are used by the ACO aided ST-MUD algorithm. Then we have $\widetilde{\mathbf{N}}_{j}=\zeta \mathbf{p}_{j}=[3.6,2.7,1.5,2.2]$. According to the traditional MUD of Alg. 1, the number of ants $\mathbf{N}_{j}$ choosing the four entries in the $j$ th column of the route-table are determined as the rounded integers of $\widetilde{\mathbf{N}}_{j}$, yielding $\mathbf{N}_{j}=[4,3,2,2]$, where the sum of the four integer elements equals to $11>\zeta=10$. In order to determine the $2^{m}$ integers in a more beneficial manner, an improved algorithm of deriving $\mathbf{N}$ from $\mathbf{p}$ is proposed for the scenarios associated with $m>1$, as detailed in the flow-chart of Alg. 2 .

In order to illustrate the advantages of the new algorithm, the same probability vector $\mathbf{p}_{j}=[0.36,0.27,0.15,0.22]$ will be used as the input vector in the following example. Firstly, the value of each element in $\mathbf{N}_{j}$ is directly given by the integer part of each element in the vector $\widetilde{\mathbf{N}}_{j}$, yielding $\mathbf{N}_{j}=[3,2,1,2]$. Then, if the sum of all the four integer elements in the vector $\mathbf{N}_{j}$ is less than $\zeta$, the $j$ th loop has to be continued, since some of the ants have not as yet carried out their decisions. More quantatively, $\sum_{i=1}^{4} N_{j i}$ ants have already made their decisions, but the remaining $\left(\zeta-\sum_{i=1}^{4} N_{j i}\right)$ ants have to make their impending choices among the four cells in the current column. The decision is made according to the value of the decimal part of the four elements of $\widetilde{\mathbf{N}}_{j}$, which is represented by the $(4 \times 1)$-element vector $\dot{\mathbf{N}}_{j}$. In the example, we have $\dot{\mathbf{N}}_{j}=[0.6,0.7,0.5,0.2]$

In order to carry out this decision, the four elements in $\dot{\mathbf{N}}_{j}$ will be ranked in ascending order according to their values. Secondly, the cell associated with the value having the highest rank will be selected as the decision of an ant randomly chosen from the remaining $\left(\zeta-\sum_{i=1}^{4} N_{j i}\right)$ ants, yielding the vector with the updated values $\mathbf{N}_{j}=[3,3,1,2]$. Then the cells will be selected from the queue constructed according to the rank of their associated value in $\dot{\mathbf{N}}_{j}$. In the given example, the output vector will then be further updated as $\mathbf{N}_{j}=[4,3,1,2]$. The selection process will not be curtailed, until all the $\zeta$ ants have made their unique decisions. As the sum of all the elements in $\mathbf{N}_{j}$ in the given example is equivalent to $\sum_{i=1}^{4} N_{j i}=$ $\zeta=10$, the process can be curtailed and the final output is the vector $\mathbf{N}_{j}=[4,3,1,2]$.

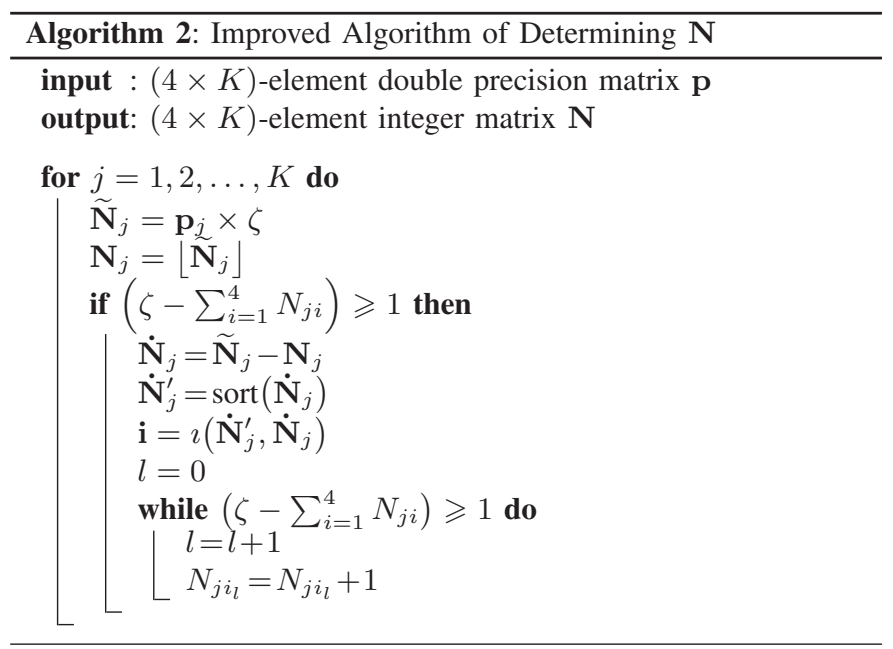

All in all, compared to the traditional ACO based MUD of Alg. 1, the improved MUD of Alg. 2 has not increased the number of cells contained by the route table nor has it increased the complexity of calculating the cellular likelihood. Additionally, the same formula was adopted by both algorithms to calculate the vector likelihood.

Despite these identical properties, the improved ACO based MUD algorithm increases the number of candidate vectors appearing in the search pool by reducing the difference between the probabilities associated with each cell within a column, while still making them equally informative. Therefore a better BER performance is expected at the price of a moderately increased complexity imposed by calculating the vector likelihood associated with the increased number of vectors. The simulation results quantifying both the achievable BER performance as a function of the complexity imposed will be detailed in the next section.

\section{Simulation Results}

Two system scenarios are considered in this section, both of which are STBC assisted two transmit antenna aided DS-CDMA systems. 
For the sake of convenience, the fully loaded DS-CDMA system supporting $K=7$ users and employing a set of $m$ sequences having a length of $N_{s}=7$ is referred to as the $m 7 K 7$ system and that supporting $K=32$ users with the aid of a set of Gold codes having a length of $N_{s}=31$ is referred to as the G31K32 system. Apart from

TABLE III: Basic simulation parameters used in Section IV.

\begin{tabular}{ll}
\hline \multicolumn{2}{c}{ ACO parameters } \\
\hline Initial pheromone & $\tau=0.01$ \\
Evaporation rate & $\rho=0.5$ \\
Maximum number of iterations & $\Xi=10$ \\
Weight of pheromone & $\alpha=1$ \\
Weight of intrinsic affinity & $\beta=6$ \\
Weight of the elite ant & $\sigma=8$ \\
\hline
\end{tabular}

using a different number of artificial ants $\zeta$, all the other parameters characterizing the ACO algorithms are identical in the traditional and in the proposed ST-MUD algorithms, which are listed in Tab. III.

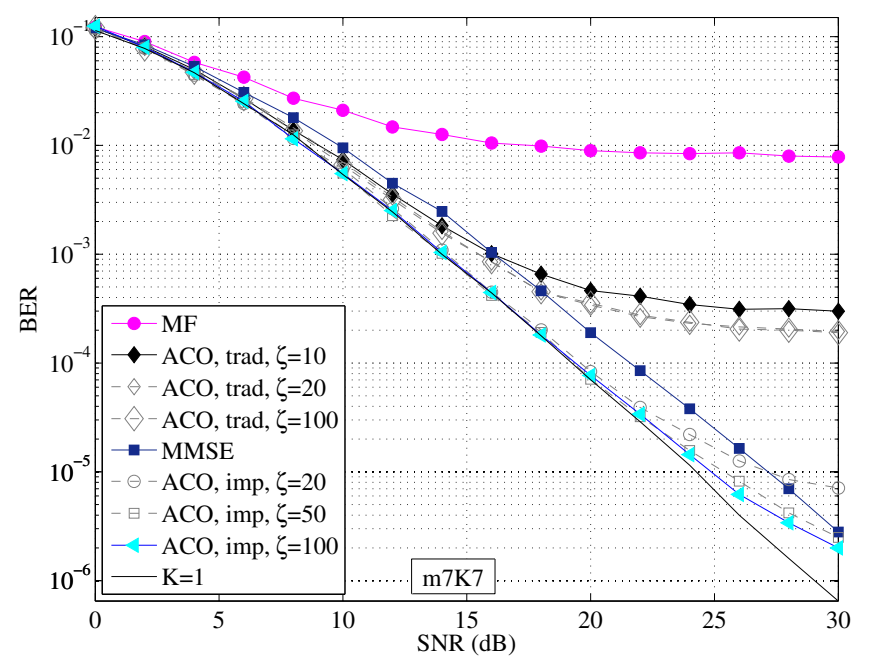

Fig. 1: Comparison of the BER performances acheived by the STBC assisted two transmit antenna rendered DS-CDMA UL employing different detection schemes, while seven users are supported by the $m$ sequence with a length of seven.

The complexity imposed is quantified in terms of the number of FLoating point Operations Per Second (FLOPS) within a STBC block duration, divided by the number of users $K$. Since both the channel quality and the AWGN vector encountered vary from block to block, the number of times we have to evaluate the $2 K$-element vector likelihood, which predetermines the total complexity imposed by the ACO algorithm is different in the consecutive block durations. Hence, to characterize the time-variant complexity of the ACO-aided STMUD algorithm more accurately, the PDF of the complexity was plotted in Fig. 2 and Fig. 4 at different SNRs ranging from OdB to $30 \mathrm{~dB}$. As seen from Fig. 2 (a) to Fig. 2 (d) and Fig. 4 (a) to Fig. 4 (d), when the SNR increases from $0 \mathrm{~dB}$ to $30 \mathrm{~dB}$, gradually decreasing complexities are imposed by both the traditional and the improved ACO ST-MUD algorithms in both the $m 7 K 7$ system and the G31K32 system.

As seen from Fig. 1, the BER performance achieved by the $m 7 K 7$ system employing the MMSE ST-MUD alogrithm is better than that of the traditional ACO ST-MUD algorithm, which is achieved as a benefit of the MMSE MUD's higher complexity, as revealed by

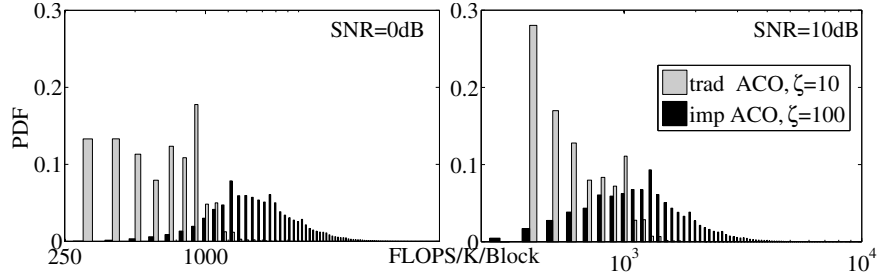

(a)

(b)

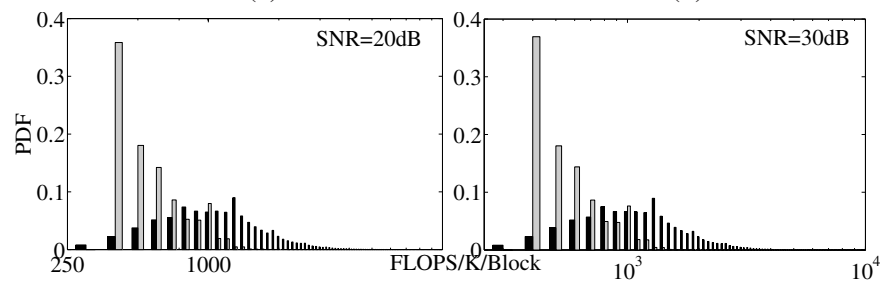

(c)

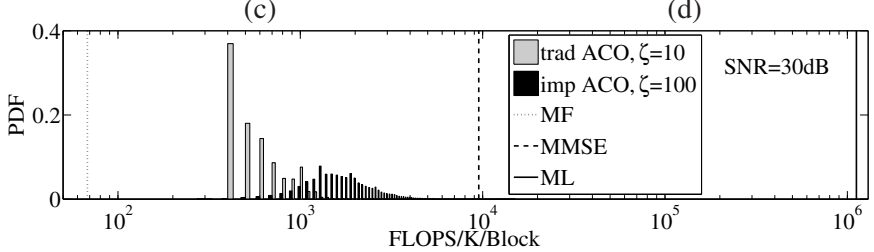

(e)

Fig. 2: PDF of quantified complexity over different Signal-to-Noise Ratio (SNR) associated with different detection schemes in the STBC assisted two transmit antenna rendered DS-CDMA UL adopting the $m$ sequence with a length of seven to support seven users.

Fig. 2 (e). The BER performance achieved by employing the traditional ACO algorithm cannot be improved by increasing the number of artificial ants, i.e. beyond $\zeta>20$. However, the proposed ACO ST-MUD algorithm shows a significant improvement of the BER performance, substantially reducing the error floor of the traditional ACO-aided MUD algorithm. Despite its improved performance, the complexity imposed by the proposed ACO algorithm is only slightly higher than that of the traditional ACO-aided ST-MUD and it is lower than that of the MMSE ST-MUD algorithm, as shown in Fig. 2 (e). Most of the time, the complexity of the proposed ACO algorithm is 1000 times lower than that of the ML algorithm.

In the G31K32 system, the BER versus SNR curve acheived by the MMSE algorithm exhibits an error floor, as shown in Fig. 3. The difference between the BER performances achieved by the traditional and the proposed ACO ST-MUD algorithm is lower in the G31K32 system compared to that in the $m 7 K 7$ system. However, the BER performance achieved by the system employing the proposed ACO algorithm is still better than that of the traditional ACO ST-MUD.

Returning to the complexity issues, as shown in Fig. 4 (e), the number of FLOPS to be carried out by the ACO ST-MUD algorithms are significantly lower than that of the MMSE ST-MUD despite their improved BER performance. Furthermore, in the $K=32$ user-scenarios both ACO algorithms impose a factor $10^{18}$ lower complexity than the ML algorithm, while allowing the G31K32 system to perform similarly to the single-user system.

\section{CONCLUSION}

We proposed a novel ACO based ST-MUD algorithm applied in the STBC assisted two transmit antenna aided DS-CDMA system. The advantages of the improved algorithm over the traditional algorithm were detailed and the BER performance provided by the improved ACO algorithm was characterized by simulation in the context of fully loaded systems employing different spreading codes. Finally, the PDF of the complexity imposed by a range of detection algorithms, including both the improved and the traditional ACO algorithms was 


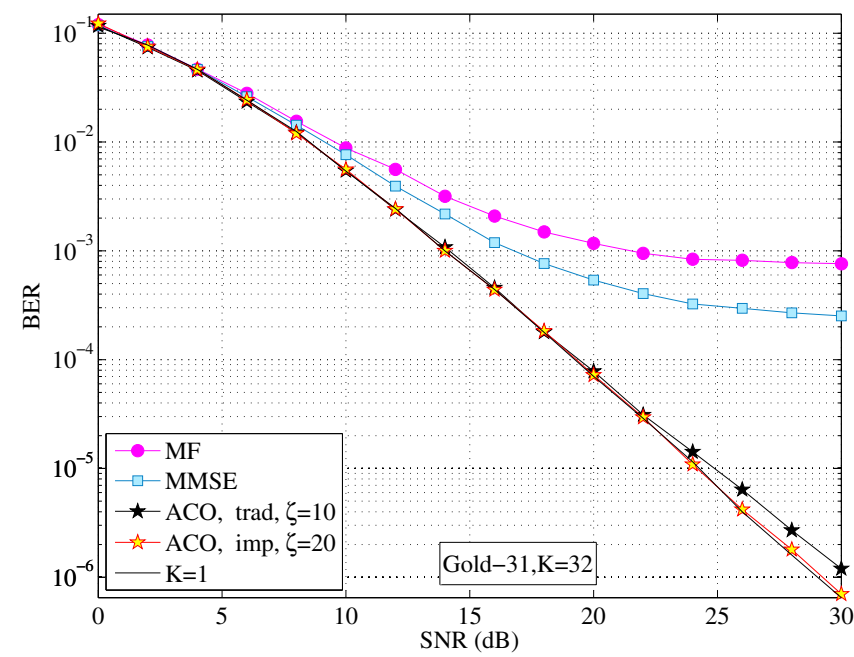

Fig. 3: Comparison of the BER performances acheived by the STBC assisted two transmit antenna rendered DS-CDMA UL employing different detection schemes, while thirty-two users are supported by Gold codes having a length of thirty-one.

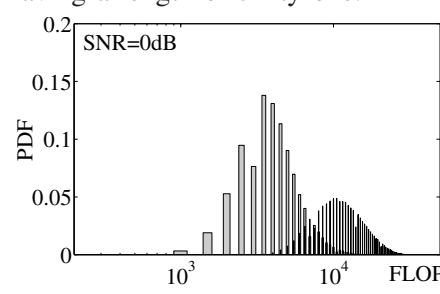

(a)

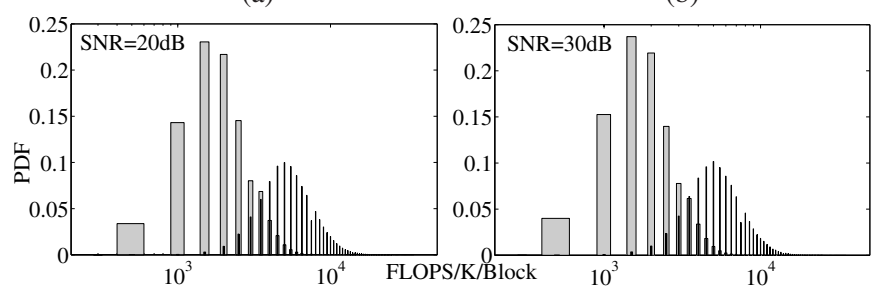

(c)

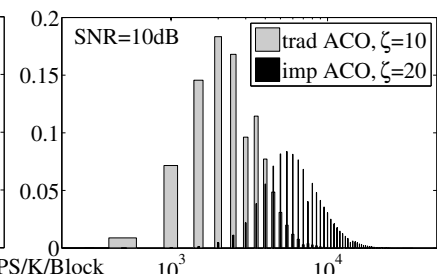

(b)

(d)

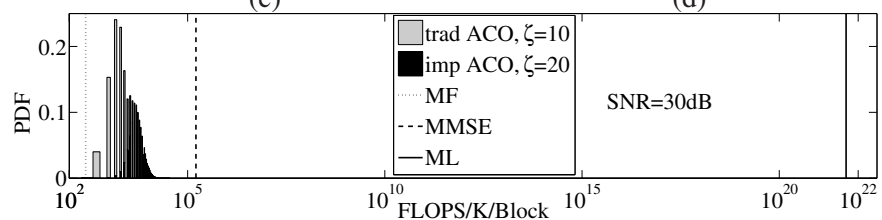

(e)

Fig. 4: PDF of quantified complexity over different SNR associated with different detection schemes in the STBC assisted two transmit antenna rendered DS-CDMA UL adopting the Gold codes with a length of thirtyone to support thirty-two users.

portrayed in Fig. 2 and Fig. 4 in order to demonstrate the advantages of the improved ACO aided ST-MUD algorithm.

\section{REFERENCES}

[1] A. Colorni, M. Dorigo and V. Maniezzo, "Distributed optimization by ant colonies," in Proceedings of the European Conference on Artificial Life, 11-13, Dec, 1991, Paris, France, pp. 134-142.

[2] T. Stützle and H.-H. Hoos,"MAX-MIN Ant system." Future Generation Computer Systems, vol. 16, pp. 889-914, June,2000.

[3] E.-G. Talbi, O. Roux, C. Fonlupt and D. Robillard, "Parallel ant colonies for the quadratic assignment problem," Future Generation Computer Systems, vol. 17, pp. 441-449, Januruary, 2001.

[4] A. Colorn, M. Dorigo, V. Maniezzo, M. Trubian, "Ant system for jobshop scheduling," JORBEL-Belgian Journal of Operations Research, Statistics and Computer Science, vol. 34, pp. 39-53, 1994.
[5] K.-M. Sim and W.-H. Sun, "Ant colony optimization for routing and load-balancing: survey and new directions," IEEE Transactions on Systems, Man and Cybernetics, Part A, vol. 33, pp. 560-572, September, 2003.

[6] S. L. Hijazi and B. Natarajan, "Near-optimal multiuser detection in asynchronous MC-CDMA via the ant colony approach," in Proceedings of the IEEE International Symposium on Wireless Pervasive Computing, 5-7, Feb, 2007, San Juan, Puerto Rico, vol. 1, pp. 274-279.

[7] S. L. Hijazi, A.J. Best B. Natarajan and S. Das, "Ant-colony based optimal MC-CDMA multiuser detector," in Proceedings of the IEEE International Conference on Wireless And Mobile Computing, Networking And Communications, vol. 1, 2005, pp. 128-132.

[8] S. L. Hijazi and B. Natarajan, "Novel low-complexity DS-CDMA multiuser detector based on ant colony optimization," in Proceedings of IEEE Vehicular Technology Conference, vol. 3, 2004, pp. 1939-1943.

[9] J.-J. Lai and J.-K. Lain, "Antenna-diversity-assisted ant-colony-based multiuser detection for DS-CDMA systems," in Proceedings of the IEEE International Workshop on Cellular Neural Networks and Their Applications, 28-30, May, 2005, Hsinchu, Taiwan, vol. 3, pp. 106-109.

[10] L. Hanzo, O. Alamri, M. E.-Hajjar and N. Wu, Near-Capacity MultiFunctional MIMO Systems USA: John Wiley and Sons, 2009.

[11] C. Xu, L.-L. Yang and L. Hanzo, "Ant-colony-based multiuser detection for MC DS-CDMA systems," in Proceedings of the IEEE Vehicular Technology Conference, 30, Sep-3, Oct, 2007, Baltimore, MD, USA, pp. $960-964$.

[12] C. Xu, B. Hu, L.-L. Yang and L. Hanzo, "Ant-colony-based multiuser detection for multi-functional antenna array assisted MC DS-CDMA systems," IEEE Transactions on Vehicular Technology, vol. 57, pp. 658663, Jan, 2008.

[13] T. C. Fogarty, "Using the genetic algorithm to adapt intelligent systems," in Proceedings of the IEE Colloquium on Symbols Versus Neurons, 1, Oct, 1990, London, UK, vol. 12,1, pp. 4/1-4/4.

[14] M. Jiang, S.-X. Ng, L. Hanzo, "Hybrid iterative multiuser detection for channel coded space division multiple access OFDM systems," IEEE Transactions on Vehicular Technology, vol. 55, pp. 115-127, Jan, 2006.

[15] L. Hanzo, L.-L. Yang, E.-L. Kuan, and K. Yen, "Single- and MultiCarrier DS-CDMA Multi-User Detection, Space-Time Spreading, Synchronisation and Standards," Chichester, UK: John Wiley and Sons, Ltd, 2003.

[16] V. Tarokh, N. Seshadri and A. R. Calderbank, "Space-time codes for high data rate wireless communication: performance criterion and code construction," IEEE Transactions on Information Theory, vol. 44 pp. 744-765, Mar, 1998.

[17] S. M. Alamouti, "A simple transmit diversity technique for wireless communications," IEEE Journal on Selected Arears of Communications, vol. 16, No. 8, pp. 1451-1458, October, 1998.

[18] J.G. Proakis, Digital Communications Fourth Edition. McGraw-Hill, 2000.

[19] T. K. Moon and W. C. Stirling, Mathematical Methods and Algorithms for Signal Processing. New Jersey, USA: Prentice Hall, 2002.

[20] S. Kay, Fundamentals of Statistical Signal Processing, Estimation The ory. New Jersey, USA: Prentice Hall, 1993.

[21] L. R. Bahl, J. Cocke, F. Jelinek, and J. Raviv, "Optimal decoding of linear codes for minimizing symbol error rate," IEEE Transactions on Information Theory, pp. 284-287, Mar, 1974.

\section{APPENDIX}

The output $\mathbf{a}^{\prime}$ of the function $\mathbf{a}^{\prime}=\operatorname{sort}(\mathbf{a})$ hosts all the elements of $\mathbf{a}$ in an ascending order according to their value. Assuming that we have $\mathbf{a}=\left[\begin{array}{llll}0.3 & 0.2 & 0.4 & 0.1\end{array}\right]$, we arrive at $\mathbf{a}^{\prime}=\operatorname{sort}(\mathbf{a})=$ $\left[\begin{array}{llll}0.4 & 0.3 & 0.2 & 0.1\end{array}\right]$.

Also assuming that $\mathbf{a}$ is $\mathbf{a}(J \times 1)$ vector, and $\mathbf{b}$ is $\mathbf{a}(J \times 1)$ vector containing all the $J$ elements in a, which are arranged in a different order than in $\mathbf{a}, \mathbf{i}=\imath(\mathbf{a}, \mathbf{b})$ becomes a $(J \times 1)$ integer vector. The $j$ th element of $\mathbf{i}, i_{j}$, is the index of the $j$ th element of $\mathbf{b}$, $b_{j}$, in the vector a. Let us give a simple example of the ' $\imath$ ' function. Then, given $\mathbf{a}=\left[\begin{array}{llll}1.0 & 0.2 & 0.3 & 2.4\end{array}\right], \mathbf{b}=\left[\begin{array}{llll}0.3 & 0.2 & 1.0 & 2.4\end{array}\right]$ and $\mathbf{i}=\imath(\mathbf{a}, \mathbf{b})$, we have $\mathbf{i}=\left[\begin{array}{llll}3 & 2 & 1 & 4\end{array}\right]$. 